\title{
On the Use of a Range Trigger for the Mars Science Laboratory Entry Descent and Landing
}

\author{
David Way \\ National Aeronautics and Space Administration \\ Langley Research Center \\ Hampton, VA 23681 \\ 757-864-8149 \\ david.w.way@nasa.gov
}

\begin{abstract}
In 2012, during the Entry, Descent, and Landing (EDL) of the Mars Science Laboratory (MSL) entry vehicle, a $21.5 \mathrm{~m}$ Viking-heritage, Disk-Gap-Band, supersonic parachute will be deployed at approximately Mach 2. The baseline algorithm for commanding this parachute deployment is a navigated planet-relative velocity trigger. This paper compares the performance of an alternative range-to-go trigger (sometimes referred to as "Smart Chute"), which can significantly reduce the landing footprint size. Numerical Monte Carlo results, predicted by the POST2 MSL POST End-to-End EDL simulation, are corroborated and explained by applying propagation of uncertainty methods to develop an analytic estimate for the standard deviation of Mach number. A negative correlation is shown to exist between the standard deviations of wind velocity and the planet-relative velocity at parachute deploy, which mitigates the Mach number rise in the case of the range trigger.
\end{abstract}

\section{Table of Contents}

1 INTRODUCTION AND BACKGROUND ........... 1

2 Monte Carlo Results................... 3

3 Theoretical Development ............. 4

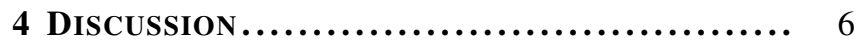

5 SUMMARY $\ldots \ldots \ldots \ldots \ldots \ldots \ldots \ldots \ldots \ldots \ldots \ldots \ldots, 8$

ACKNOWLEDGEMENTS ..................... 8

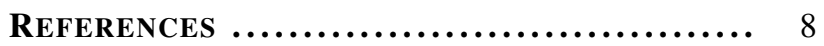

BIOGRAPHY

\section{NOMENCLATURE}

$\begin{array}{ll}\mu & \text { statistical mean } \\ \rho & \text { atmospheric density } \\ \rho_{x y} & \text { correlation coefficient } \\ \sigma & \text { standard deviation } \\ \sigma^{2} & \text { statistical variance } \\ c_{s} & \text { speed of sound } \\ M & \text { Mach number } \\ q & \text { dynamic pressure } \\ V_{A} & \text { atmospheric-relative velocity } \\ V_{R} & \text { planet-relative velocity }\end{array}$

${ }^{1}$ U.S. Government work not protected by U.S. copyright.

2 IEEEAC Paper \#1142, Final Version 1, Updated January 5, 2011.

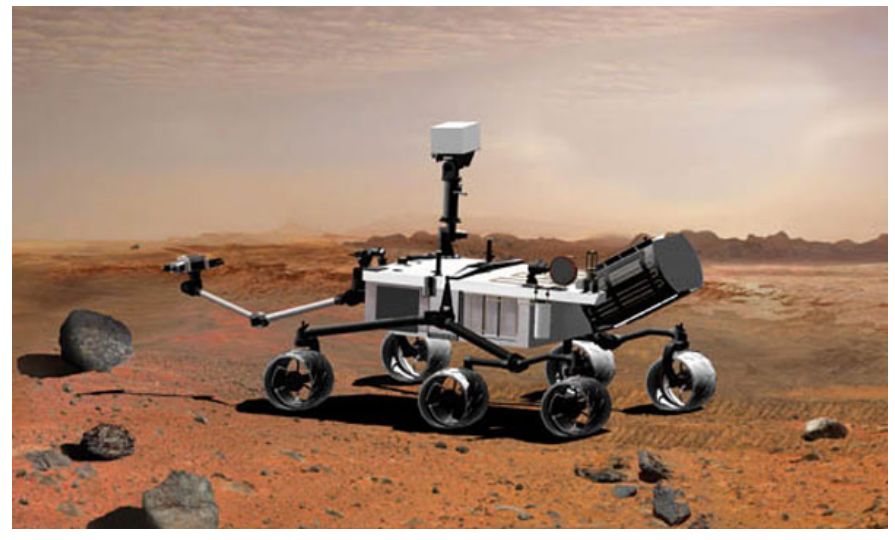

Figure 1. Artist concept of Mars Science Laboratory rover, named Curiosity, exploring Mars.

$V_{W} \quad$ wind velocity

DGB Disk-Gap-Band Parachute

EDL Entry, Descent, and Landing

IMU Inertial Measuring Unit

MER Mars Exploration Rover

MOLA Mars Orbiting Laser Altimeter

MSL Mars Science Laboratory

POST2 Program to Optimize Simulated Trajectories II

TPS Thermal Protection System

\section{INTRODUCTION AND BACKGROUND}

\section{Mars Science Laboratory}

Scheduled to launch in the 2011 Type-I Mars opportunity, Mars Science Laboratory (MSL) will be the next robotic mission to explore the surface of the red planet under NASA's Mars Exploration Program. Building on the success of the twin Mars Exploration Rover (MER) rovers, Spirit and $O p$ portunity, which landed in 2004, the $M S L$ rover, named $\mathrm{Cu}$ riosity, will assess the planet's present and past habitability. Armed with a suite of state-of-the-art scientific instruments, Curiosity, shown in Figure 1, will collect dozens of soil and rock samples and analyze them in an on-board laboratory for the presence of biomarkers, the chemical building blocks of life.

Designing an Entry, Descent, and Landing (EDL) system to 


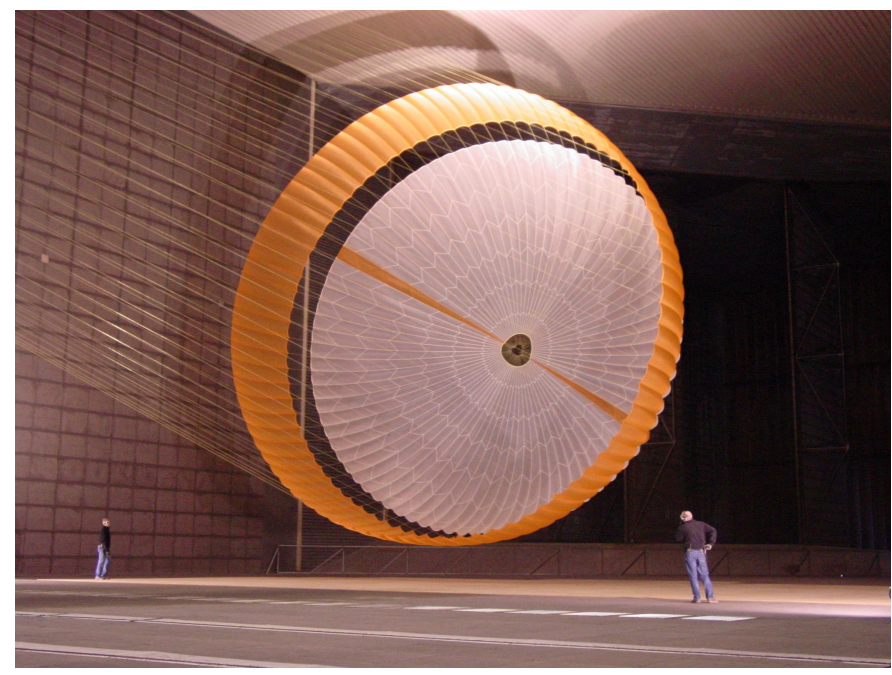

Figure 2. Mars Science Laboratory DGB parachute undergoing full-scale wind-tunnel testing.

deliver such a large and capable rover safely to a scientifically compelling site, which is rich in minerals likely to trap and preserve biomarkers, presents a myriad of engineering challenges. Not only is the payload mass significantly larger than all previous Mars missions, the delivery accuracy and terrain requirements are also more stringent. In August of 2012, MSL will enter the Martian atmosphere with the largest aeroshell ever flown to Mars, fly the first guided lifting entry at Mars, generate a higher hypersonic lift-to-drag ratio than any previous Mars mission, and decelerate behind the largest supersonic parachute ever deployed at Mars. The MSL EDL system will also, for the first time ever, softly land Curiosity directly on her wheels, ready to explore the planet's surface.

\section{Parachute Decelerators for Mars}

Since the first Viking landing in 1976, the super-sonic deployment of a parachute has been a critical event in all Mars EDL systems. This is because at Mars, due to the planet's thin atmosphere, only entry systems with ballistic coefficients below about $50 \mathrm{~kg} / \mathrm{m}^{2}$ have the ability to deliver payloads to subsonic terminal velocities [1]. For $M S L$, a blunt aeroshell, with a ballistic coefficient of approximately $140 \mathrm{~kg} / \mathrm{m}^{2}$, is first used to slow the vehicle from hypersonic entry velocities as high as $6 \mathrm{~km} / \mathrm{s}$ down to low super-sonic speeds, near $400 \mathrm{~m} / \mathrm{s}$. At that point, the $21.5 \mathrm{~m}$ diameter Disk-Gap-Band Parachute Decelerator System (PDS), shown in Figure 2, is then used to reduce the ballistic coefficient to approximately $15 \mathrm{~kg} / \mathrm{m}^{2}$. The parachute continues slowing the vehicle below Mach 1 to a sub-sonic terminal velocity of approximately $100 \mathrm{~m} / \mathrm{s}$.

Because of the importance of the parachute deployment event, parachute failure is a key risk considered in Mars EDL system design. Higher Mach numbers and dynamic pressure during parachute inflation put the parachute at a higher risk of failure due to three factors: (1) higher dynamic pressures result in higher structural loads on the parachute; (2) higher Mach numbers result in increased aerothermal heating of parachute structure, which can reduce material strength; and (3) at Mach numbers above Mach 1.5, DGB parachutes exhibit an instability, known as areal oscillations, which result in multiple partial collapses and violent re-inflations. The chief concern with high Mach number deployments, for parachute deployments in regions where the heating is not a driving factor, is therefore, the increased exposure to areal oscillations.

The Viking parachute system was qualified to deploy between Mach 1.4 and 2.1, and a dynamic pressure between 250 and $700 \mathrm{~Pa}$ [1]. However, Mach 2.1 is not a hard limit for successfully operating DBG parachutes at Mars and there is very little flight test data above Mach 2.1 with which to quantify the amount of increased EDL system risk. Figure 3 shows the relevant flight tests and flight experience in the region of the planned MSL parachute deploy. While parachute experts agree that higher Mach numbers result in a higher probability of failure, they have different opinions on where the limit should be placed. For example, Gillis [5] has proposed an upper bound of Mach 2 for parachute aerodynamic decelerators at Mars. However, Cruz [3] places the upper Mach number range somewhere between two and three.

This presents a challenge for EDL system designers, who must then weigh the system performance gains and risks associated with deploying the parachute earlier, at both higher altitudes and Mach numbers, against a very real, but not well quantified, probability of parachute failure. It is clear that deploying a DGB at Mach 2.5 or 3.0 represents a significant increase in risk over an inflation at Mach 2.0. However, it is not clear how much additional risk is encumbered by deploying the parachute at 2.25 instead of 2.05. This is especially true for Mars EDL in light of the extremely large uncertainties in the flight environment, especially atmospheric density and winds, that result in very large uncertainties in Mach number.

\section{Parachute Deployment Algorithms}

Previous missions have utilized various methods for triggering parachute deployment. Though the parachute qualification has been stated in terms of Mach number and dynamic pressure, no previous mission has had the ability to directly measure either of these quantities. Therefore, all missions have had to rely on proxy measurements of other states in order to infer whether or not conditions were safe for deploying the parachute. Viking used a radar altimeter measurement to trigger this critical event [6]. Mars Pathfinder [8] and MER [4] both used triggers based on sensed-acceleration measurements, provided by the on-board Inertial Measurement Unit (IMU), to proxy dynamic pressure, though their algorithms differed. Mars Phoenix Lander, on the other hand, used a navigated velocity trigger to proxy Mach number.

When originally proposed, MSL (known then as Mars Smart Lander) featured a range trigger as part of the Apollo-heritage entry guidance system, often referred to as the Smart Chute 


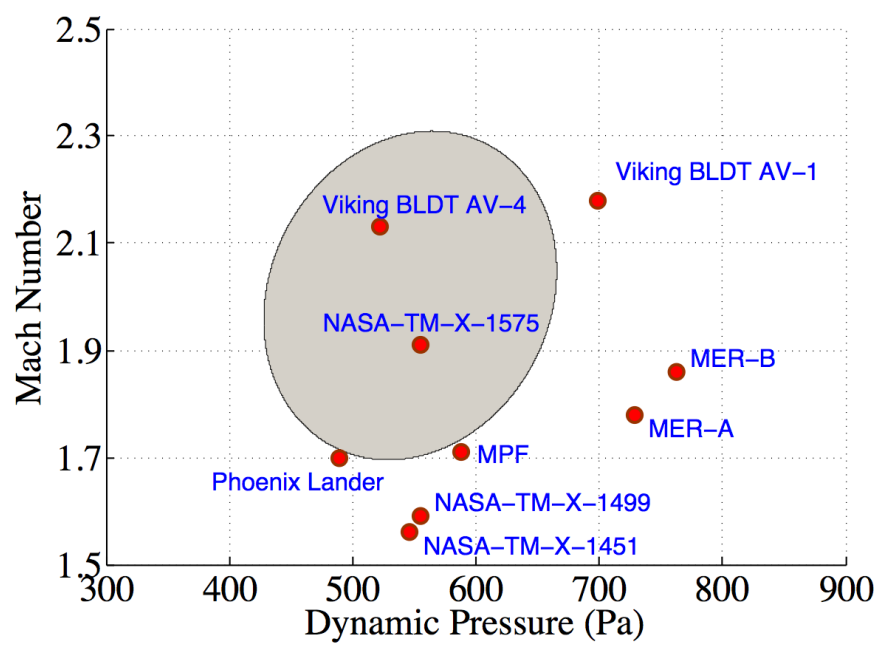

Figure 3. Relevant test and flight experience of supersonic Disk-Gap-Band parachutes in the region of $M S L$ parachute deployment (shaded region).

algorithm. To minimize the parachute deploy footprint, the parachute was to be deployed on a command from the entry guidance when the estimated range-to-go to the target was minimized [7]. As an additional safety measure, the Smart Chute algorithm also included navigated velocity limits. These limits were used in order to protect the parachute against either excessively high dynamic pressures or excessively low deployment altitudes [2]. Above the high velocity set-point, parachute deploy was inhibited. Below the low velocity limit, parachute deploy was triggered, regardless of range-to-go.

Eventually, the Smart Chute range trigger was dropped from the MSL baseline in-favor of a velocity trigger. The rationale for this decision was to maximize the altitude performance of the system, which was being strained at that time by rapid mass growth of the rover and a very challenging altitude requirement for demonstrating the capability to land as high as $+2.0 \mathrm{~km}$ above the MOLA reference areoid. It was argued at the time, that due the monotonically decreasing altitude and velocity just prior to parachute deploy, the upper velocity limit of the Smart Chute represented the earliest, and therefore highest, deployment condition that was considered safe. Replacing the Smart Chute trigger with a pure velocity trigger, operated at the same set-point as the upper velocity limit, would maximize the parachute deploy altitude, while maintaining the same level of risk to the parachute.

\section{Study Motivation}

As stated, the switch from a range trigger to a velocity trigger had been argued for the maximization of parachute deploy altitude. Though the project would eventually receive some relief from the $+2.0 \mathrm{~km}$ altitude requirement, a premium on altitude performance existed for quite some time. Concurrently, however, the project had initiated a series of Landing Site Workshops, open to the scientific community, for the pur-
Table 1. MSL Candidate Landing Sites

\begin{tabular}{|c|c|c|c|}
\hline $\begin{array}{c}\text { Site } \\
\text { Name }\end{array}$ & $\begin{array}{c}\text { Lat. } \\
(\mathrm{deg})\end{array}$ & $\begin{array}{c}\text { Lon. } \\
(\mathrm{deg})\end{array}$ & $\begin{array}{c}\text { Elevation } \\
(\mathrm{km})\end{array}$ \\
\hline Mawrth Valis & $24.01^{\circ} \mathrm{N}$ & $341.03^{\circ} \mathrm{E}$ & -2.25 \\
Gale Crater & $4.49^{\circ} \mathrm{S}$ & $137.42^{\circ} \mathrm{E}$ & -4.45 \\
Eberswalde Crater & $23.86^{\circ} \mathrm{S}$ & $326.73^{\circ} \mathrm{E}$ & -1.45 \\
Holden Crater & $26.37^{\circ} \mathrm{S}$ & $325.10^{\circ} \mathrm{E}$ & -1.94 \\
\hline
\end{tabular}

pose of proposing and selecting possible landing sites. While many sites were initially proposed at the first of these workshops, the outcome of the 4th Landing Site Workshop in 2008 was a list of four candidate sites, listed in Table 1. Of the four final sites, Eberswalde Crater has the highest elevation at $-1.45 \mathrm{~km} \mathrm{MOLA}$, which is significantly below the altitude capability of the system (estimated to be somewhere around $0 \mathrm{~km} \mathrm{MOLA).} \mathrm{These} \mathrm{lower} \mathrm{site} \mathrm{altitudes} \mathrm{have} \mathrm{improved} \mathrm{EDL}$ timeline margins significantly compared to the time when the parachute deploy trigger was changed. In light of this reduced premium on altitude, this study sought to re-evaluate the merits of a range trigger relative to the baseline velocity trigger.

\section{Monte Carlo Results}

A side-by-side comparison of a range trigger and velocity trigger was conducted for MSL. The purpose of this comparison was to evaluate the relative performance of the range trigger relative to the baseline velocity trigger. A single hybrid velocity-range trigger was developed that is capable of emulating either velocity or range triggers by appropriate choice of parameters. This trigger works by specifying a linear switching curve in velocity-range space. The algorithm triggers parachute deploy when the velocity drops below the switching curve for the given range to target. A horizontal switching curve, therefore, produces a pure velocity trigger, while a vertical switching curve, on the other hand, produces a pure range trigger.

For each trigger a 6-DoF Monte Carlo analysis was performed using the 08-GAL-06 MSL POST2 end-to-end EDL performance simulation. The two triggers were each independently tuned to produce the same nominal parachute deploy at Mach 2.0, as was the standard project procedure for running Monte Carlos. It was expected that the results would show a smaller parachute deploy footprint for the range trigger at the expense of reduced altitude performance and increased deploy Mach number.

Figure 4 shows the expected reduction in the $99.5 \%$-tile footprint ellipse. In this case, the ellipse was reduced from 16.7 by $7.5 \mathrm{~km}$ for the velocity trigger to 7.7 by $4.1 \mathrm{~km}$ for the range trigger, a $75 \%$ reduction in area. However, the expected altitude loss and Mach increase were not observed.

Table 2 contains a summary of Monte Carlo results for the 

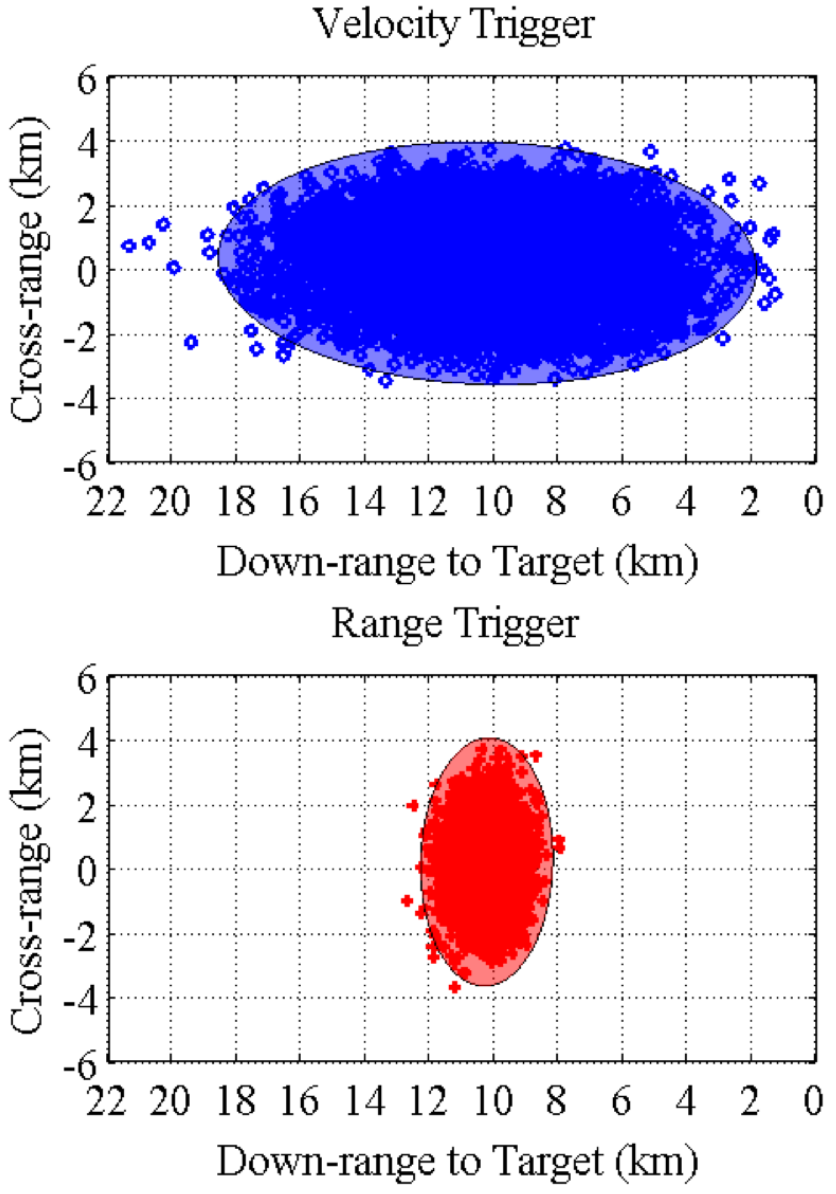

Figure 4. Comparison of Parachute Deploy Footprints.

velocity and range triggers. The mean values in Table 2 are similar, by design, because both triggers were tuned for the same nominal parachute deploy Mach number. However, even though the standard deviation of planet-relative velocity is nearly 5.5 times larger for the range trigger, the standard deviation of atmospheric-relative velocity is only $24 \%$ higher, and the standard deviation of Mach number increased by only 0.006 . This results in an increase of only 0.033 in the 99th percentile of Mach number. Figure 5 compares the histograms for parachute deploy Mach number. Strictly speaking, even this slight increase in Mach number represents some higher risk to the parachute system. Practically, however, the amount of additional risk is negligible and impossible to quantify given the limited test data available. Therefore, the nominal deploy Mach number for the range trigger was not lowered to bring the 99\%-tile Mach in agreement with the velocity trigger results.

Finally, because the mean deploy altitudes were identical (within $10 \mathrm{~m}$ ) and because the standard deviation of altitude was slightly smaller for the range trigger, the range trigger showed a $218 \mathrm{~m}$ increase in 1\%-tile altitude over the velocity trigger. This result was contrary to the expected altitude loss, but related to the negligible increase in Mach number. An
Table 2. Monte Carlo Results

\begin{tabular}{|c|c|c|c|}
\hline Parameter & Units & $\begin{array}{l}\text { Velocity } \\
\text { Trigger }\end{array}$ & $\begin{array}{l}\text { Range } \\
\text { Trigger }\end{array}$ \\
\hline \multicolumn{4}{|l|}{ Mean Value } \\
\hline Planet-rel. velocity & $\mathrm{m} / \mathrm{s}$ & 463.8 & 464.2 \\
\hline Atm.-relative velocity & $\mathrm{m} / \mathrm{s}$ & 447.2 & 447.3 \\
\hline Mach number & - & 2.002 & 2.001 \\
\hline Dynamic pressure & $P a$ & 546.7 & 546.7 \\
\hline Parachute opening load & $1000 \mathrm{lbs}$. & 47.64 & 47.67 \\
\hline Altitude wrt. MOLA & $\mathrm{km}$ & 10.084 & 10.073 \\
\hline \multicolumn{4}{|l|}{ Standard Deviation } \\
\hline Planet-rel. velocity & $\mathrm{m} / \mathrm{s}$ & 3.0 & 16.2 \\
\hline Atm.-relative velocity & $\mathrm{m} / \mathrm{s}$ & 13.0 & 16.1 \\
\hline Mach number & - & 0.096 & 0.102 \\
\hline Dynamic pressure & $P a$ & 37.2 & 39.5 \\
\hline Parachute opening load & $1000 \mathrm{lbs}$. & 3.57 & 3.43 \\
\hline Altitude wrt. MOLA & $\mathrm{km}$ & 1.129 & 1.077 \\
\hline \multicolumn{4}{|l|}{ 99 $9^{\text {th }}$ Percentile } \\
\hline Planet-rel. velocity & $\mathrm{m} / \mathrm{s}$ & 470.6 & 499.1 \\
\hline Atm.-relative velocity & $\mathrm{m} / \mathrm{s}$ & 475.1 & 483.6 \\
\hline Mach number & - & 2.221 & 2.254 \\
\hline Dynamic pressure & $P a$ & 639.9 & 647.1 \\
\hline Parachute opening load & $1000 \mathrm{lbs}$. & 56.92 & 56.28 \\
\hline \multicolumn{4}{|l|}{$1^{1^{s t}}$ Percentile } \\
\hline Altitude wrt. MOLA & $\mathrm{km}$ & 7.343 & 7.561 \\
\hline
\end{tabular}

appreciable increase in 99\%-tile Mach number would have forced a reduction in mean Mach number, resulting in a mean altitude loss of approximately $85 \mathrm{~m}$ per 0.01 Mach number decrease.

These Monte Carlo results suggested a very favorable trade to adopt the range trigger, but more analysis was needed to explain why they were contrary to the expectations of the EDL team. However, because Monte Carlo is a numerical analysis, it is very difficult, if not impossible, to explain why these results differed so dramatically from expectation. Therefore, an analytical approach is developed in the following section to attempt to explain the observed numerical results.

\section{THEORETICAL DEVELOPMENT}

\section{Analytical Approach}

We desire an analytical expression which relates random variables at the instant of parachute deploy to the resulting distribution in Mach number. To simplify the analysis, we will assume that all random variables are normally distributed. 

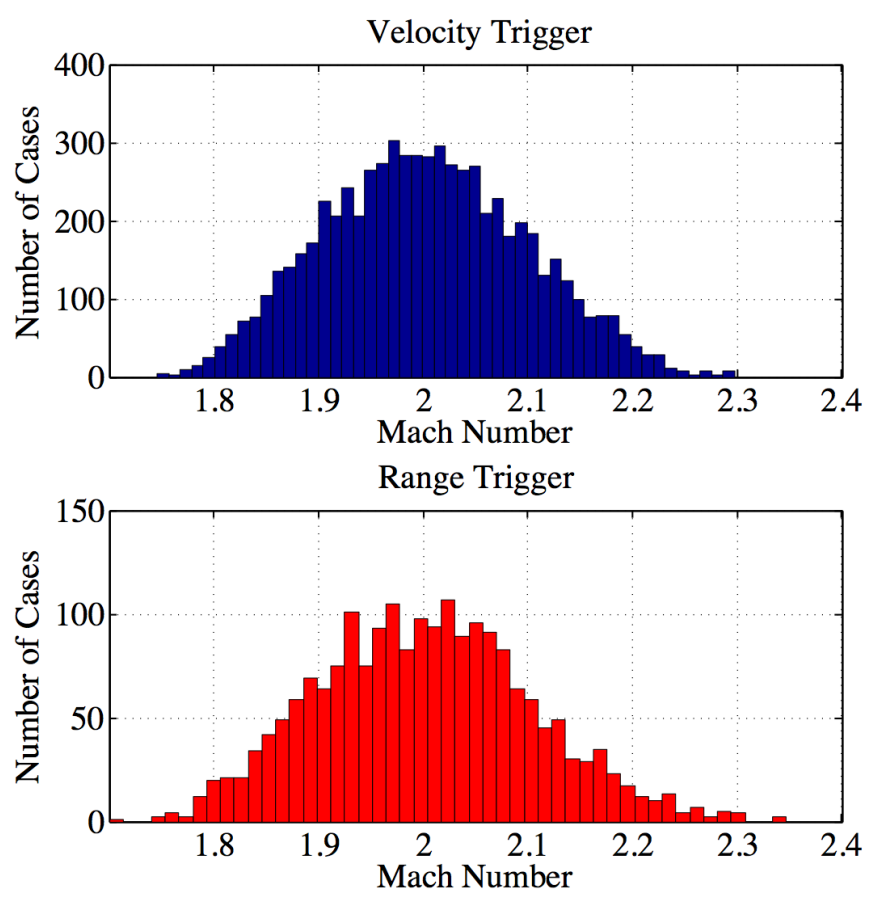

Figure 5. Comparison of Mach Number at Parachute Deploy.

Though this assumption is not correct in general, the particular random variables of interest from the numerical Monte Carlo simulation are approximated very well by Gaussian distributions. Furthermore, we will focus on estimating the standard deviation, rather than the mean, since the later is a tunable parameter.

We wish to write, therefore, the standard deviation of Mach number, $\sigma_{M}$, as a function of the standard deviations of relative velocity $\left(V_{R}\right)$, wind velocity $\left(V_{W}\right)$, and speed of sound $\left(c_{s}\right), \sigma_{V_{R}}, \sigma_{V_{W}}$, and $\sigma_{c_{s}}$ respectively. We see from the scalar definition of atmospheric-relative velocity (Eqn. 1) and Mach number (Eqn. 2) that these flight parameters are the constituents of Mach number. Here $V_{A}$ is defined as a scalar sum, rather than a strict vector summation, so that $V_{W}$ is more correctly the projection of the wind vector on the planet-relative velocity vector, with the convention of a positive value indicating a head-wind.

$$
\begin{gathered}
V_{A}=V_{R}+V_{W} \\
M=\frac{V_{A}}{c_{s}}
\end{gathered}
$$

\section{Propagation of Uncertainty}

We apply propagation of uncertainty to the above definitions to get expressions for the standard deviations of atmosphericrelative velocity and Mach number. We assume that distributions of the flight conditions, $V_{R}, V_{W}$, and $c_{s}$, are either known or specified and that they are normally distributed, such that $V_{R}=N\left(\mu_{V_{R}}, \sigma_{V_{R}}^{2}\right), V_{W}=N\left(\mu_{V_{W}}, \sigma_{V_{W}}^{2}\right)$, and $c_{s}=N\left(\mu_{c_{s}}, \sigma_{c_{s}}^{2}\right)$.

Note that two of these random variables, wind velocity and speed of sound, are environmental parameters. Their distributions are outputs of the simulation's atmosphere model in the region of the parachute deploy point and are independent of the trigger algorithm. For $M S L$, the uncertainty in these parameters is determined statistically by sampling multiple sols and local solar times predicted by meso-scale computer simulations of the Martian atmosphere. The third random variable, planet-relative velocity, is a result of integrating the equations of motion in time from the initial state to the instant of parachute deploy. Since different algorithms will trigger parachute deploy at different times, the uncertainty in $V_{R}$ is a characteristic of the algorithm employed.

Equation 1 may be recognized as a linear combination of normal distributions. Therefore, the result is known to be also normally distributed, $V_{A}=N\left(\mu_{V_{A}}, \sigma_{V_{A}}^{2}\right)$, with a standard deviation $\sigma_{V_{A}}$ given by Equation 3. Note that while the distributions $V_{R}$ and $V_{W}$ are assumed to be normal distributions, they are not necessarily independent. The angle, $\theta$, in the third term of the radical is a correlation angle, which is related to the correlation coefficient, $\rho_{x y}$, between the two random variables. A correlation angle of $\theta=0$ is equivalent to a correlation coefficient of $\rho_{x y}=0$, which implies that there is no linear correlation between the variables. Likewise, a correlation angle, $\theta=\frac{\pi}{2}$, is equivalent to a correlation coefficient of $\rho_{x y}=1$, which implies a direct one-to-one relationship between the variables.

$$
\sigma_{V_{A}}=\sqrt{\sigma_{V_{R}}^{2}+\sigma_{V_{W}}^{2}+2 \sigma_{V_{R}} \sigma_{V_{W}} \sin \theta}
$$

Similarly, by taking the natural logarithm of Equation 2, Equation 4 may also be recognized as a linear combination of normal distributions, if one assumes that the logarithm of a normal distribution is also Gaussian. This is approximately true if the random variable has a non-zero mean and a standard deviation that is small compared to that mean. Equation 5 relates the standard deviation of the natural logarithm of a random variable to the random variable's standard deviation. In practice, the error in this approximation is typically less than $1 \%$ for random variables that have a mean to standard deviation ratio $\left(\mu_{X} / \sigma_{X}\right)$ greater than about 12 .

$$
\ln M=\ln V_{A}-\ln c_{s}
$$

$$
\sigma_{\ln (X)} \approx \frac{1}{\left|\mu_{X}\right|} \sigma_{X}
$$

Using this approximation results in Equation 6 for the standard deviation of $\ln M$. Again, the random variables are all 
assumed to be normally distributed, but not necessarily uncorrelated. The angle, $\alpha$, in the third term of the radical in Eqn. 6 is the correlation angle between $\ln V_{A}$ and $\ln c_{s}$.

$$
\sigma_{\ln M}=\sqrt{\sigma_{\ln V_{A}}^{2}+\sigma_{\ln c_{s}}^{2}+2 \sigma_{\ln V_{A}} \sigma_{\ln c_{s}} \sin \alpha}
$$

Finally, substituting Eqn. 3 into Eqn. 6 and using the approximation, Eqn 5, results in the desired expression for $\sigma_{M}$. This expression, Equation 7, predicts the standard deviation of Mach number as a function of the three mean values $\left(\mu_{V_{R}}\right.$, $\mu_{V_{W}}$, and $\left.\mu_{c_{s}}\right)$, three standard deviations $\left(\sigma_{V_{R}}, \sigma_{V_{W}}\right.$, and $\left.\sigma_{c_{s}}\right)$, and the two correlation angles $(\theta$ and $\alpha)$.

$$
\begin{gathered}
\sigma_{M} \approx \mu_{M}\left\{\begin{array}{c}
\frac{\sigma_{V_{R}}^{2}}{\left(\mu_{V_{R}}+\mu_{V_{W}}\right)^{2}} \\
+\frac{\sigma_{V_{W}}^{2}}{\left(\mu_{V_{R}}+\mu_{V_{W}}\right)^{2}} \\
+\frac{\sigma_{c_{s}}^{2}}{\mu_{c_{s}}^{2}} \\
+A \sin \theta \\
+B \sin \alpha
\end{array}\right\}^{\frac{1}{2}} \\
A=\frac{2 \sigma_{V_{R}} \sigma_{V_{W}}}{\left(\mu_{V_{R}}+\mu_{V_{W}}\right)^{2}} \\
B=2 \frac{\left(\sigma_{V_{R}}^{2}+\sigma_{V_{W}}^{2}+2 \sigma_{V_{R}} \sigma_{V_{W}} \sin \theta\right)^{\frac{1}{2}}}{\left(\mu_{V_{R}}+\mu_{V_{W}}\right)} \frac{\sigma_{c_{s}}}{\mu_{c_{s}}}
\end{gathered}
$$

The first term in the radical of Eqn. 7 is the planet-relative velocity term. The magnitude of the variance of $V_{R}$ is not only dependent on the integrated effect of the dispersions leading up to parachute deploy, but also on the particular triggering algorithm, since these will affect the time at which deployment occurs. The second and third terms are the wind velocity term and speed-of-sound term, respectively, which are independent of the triggering logic. The fourth term is the wind correlation, which will be discussed in more detail in the following section. This is the statistical correlation between the wind velocity and the planet-relative velocity at the moment of parachute deploy. The last term is the speed-ofsound correlation.

\section{Discussion}

\section{Comparison of Results}

The analytic expressions derived in the previous sections were validated by comparison with the Monte Carlo numerical results. Three Monte Carlo results are compared: a velocity trigger, a hybrid trigger, and a range trigger. For the hybrid trigger case, the switching curve was set with a finite, positive slope to produce intermediate results that were neither a pure velocity trigger nor a pure range trigger, but roughly halfway between the two. Figure 6 plots the resulting dispersions in the velocity-range plane and shows the primary effect of varying the trigger, which is to slice the corridor of incoming trajectories at a different angle changing the projections

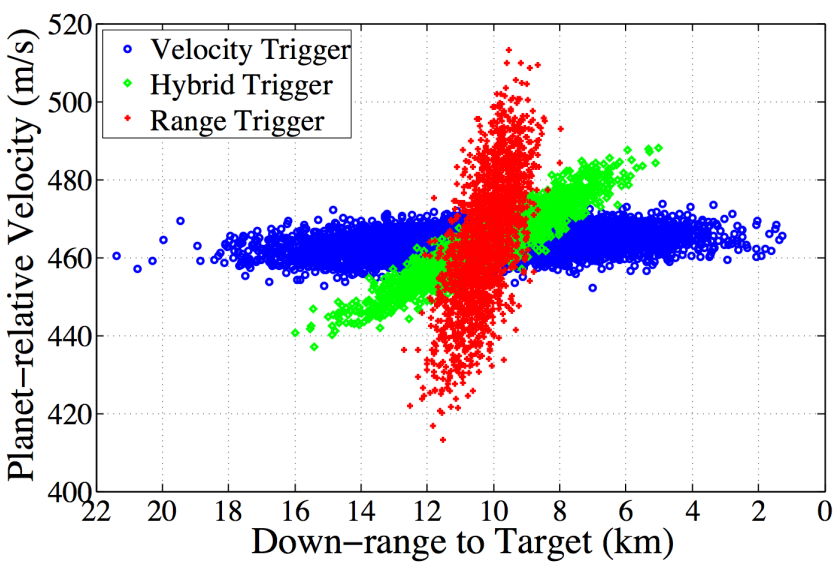

Figure 6. Parachute Deploy Conditions, Velocity vs. Range.

of the dispersions in the altitude and velocity axes.

Statistics from the Monte Carlos, presented in Table 3, were collected on flight parameters $V_{R}, V_{W}$, and $c_{s}$ at parachute deploy as well as the two correlation angles, $\theta$ and $\alpha$. These correlation angles are found from the arcsine of the correlation coefficients of the Monte Carlo data. These values were used to estimate $\sigma_{M}$ (Equation 7). As shown in Table 4, these analytic estimates compared very well with the numerical results with a maximum error of $0.3 \%$ of the actual result.

Table 3 shows that $\sigma_{V_{R}}$ progressively increases from $3.0 \mathrm{~m} / \mathrm{s}$ for the velocity trigger to $16.2 \mathrm{~m} / \mathrm{s}$ for the range trigger. The wind dispersions, $\sigma_{V_{W}}$ are consistent, as expected, since it is an environmental parameter. The slight decrease in $\sigma_{c_{s}}$ is due to altitude differences. Note that the wind correlation angle, $\theta$, is negative and that its value is the same for the hybrid and range triggers and only a few degrees different for the velocity trigger. This correlation is discussed in the following section.

Table 3. Parachute Deploy Flight Conditions

\begin{tabular}{|l|c|c|c|c|}
\hline Parameter & Units & $\begin{array}{c}\text { Velocity } \\
\text { Trigger }\end{array}$ & $\begin{array}{c}\text { Hybrid } \\
\text { Trigger }\end{array}$ & $\begin{array}{c}\text { Range } \\
\text { Trigger }\end{array}$ \\
\hline \hline Mean Value \\
\hline \hline Planet-rel. vel. & $m / s$ & 463.8 & 463.7 & 464.2 \\
Wind velocity & $m / s$ & -16.6 & -16.8 & -16.9 \\
Speed-of-sound & $m / s$ & 223.7 & 223.8 & 223.8 \\
\hline \hline \multicolumn{5}{|l}{ Standard Deviation } \\
\hline \hline Planet-rel. vel. & $m / s$ & 3.0 & 8.5 & 16.2 \\
Wind velocity & $m / s$ & 13.6 & 13.7 & 13.7 \\
Speed-of-sound & $m / s$ & 8.0 & 7.9 & 7.8 \\
\hline \hline Correlation & \multicolumn{5}{l}{} \\
\hline \hline$\theta\left(V_{R}, V_{W}\right)$ & $d e g$ & -18.0 & -25.6 & -25.4 \\
$\alpha\left(V_{A}, c_{s}\right)$ & $d e g$ & 4.6 & 3.2 & 1.7 \\
\hline
\end{tabular}


Table 4. Comparison of Analytic and Numeric Results for Standard Deviation of Mach Number

\begin{tabular}{|l|c|c|}
\hline$\sigma_{M}$ & $\begin{array}{c}\text { Analytic } \\
\text { Estimate }\end{array}$ & $\begin{array}{c}\text { Numerical } \\
\text { Result }\end{array}$ \\
\hline \hline Velocity Trigger & 0.0957 & 0.0959 \\
Hybrid Trigger & 0.0931 & 0.0933 \\
Range Trigger & 0.1019 & 0.1022 \\
\hline
\end{tabular}

\section{Wind Correlation}

Because the velocity trigger has, by design, a negligible standard deviation of planet-relative velocity (the triggering parameter), the variance in atmospheric-relative velocity is due solely to the variance in the wind at parachute deploy. In contrast, the range trigger has a much larger variance in planetrelative velocity, which is traded, again by design, for a small variance in range. Consequently, the range trigger, having the same wind dispersions as the velocity trigger, should be expected to have a much larger variance in atmospheric-relative velocity, and therefore, Mach number. However, this result is not seen in the Monte Carlo analysis.

The reason for this is the negative correlation angle $(\theta)$ between the planet-relative velocity and the wind velocity at parachute deploy, shown in Table 3. Physically, this correlation exists because the drag, experienced during entry, is a function of the atmospheric-relative velocity, and therefore, wind. If the entry vehicle is experiencing a head wind (positive wind speed by our convention), the atmospheric-relative velocity and drag will be higher than a no-wind case. Therefore, the head-wind case will arrive at the parachute deploy point at a lower planet-relative velocity. Conversely, if the entry vehicle experiences a tail wind (negative wind), the atmospheric-relative velocity and drag will be lower, resulting in a higher planet-relative velocity at parachute deploy.

Graphically, the effect of this wind correlation on $\sigma_{V_{A}}$ can be seen in the example plotted in Figure 7. Here the blue vector (OA) has been drawn along the horizontal axis with a magnitude equal to the standard deviation of the wind speed, $\sigma_{V_{W}}$ $=15 \mathrm{~m} / \mathrm{s}$, in this example. The standard deviation of planetrelative velocity (OB) is drawn in green, $\sigma_{V_{R}}=20 \mathrm{~m} / \mathrm{s}, \theta$ $=-22 \mathrm{deg}$. The angle between vectors $\mathrm{OA}$ and $\mathrm{OB}$ is determined by the correlation between the random variables $V_{R}$ and $V_{W}$. The red vector (OC) is the standard deviation of atmospheric-relative velocity, $\sigma_{V_{A}}=20 \mathrm{~m} / \mathrm{s}$, resulting from the vector addition of OA and OB. Because of the negative correlation, $\sigma_{V_{A}}$ is significantly smaller than the $25 \mathrm{~m} / \mathrm{s}$ that would be expected for uncorrelated random variables.

For all hybrid triggers, the magnitude of $\sigma_{V_{R}}$ is smaller, but the correlation angle, $\theta$ is the same. This is because the correlation angle is a property of the simulation, independent of the trigger, resulting from the integration of the winds prior to parachute deploy. Therefore, the chord AC in Figure 7 repre-

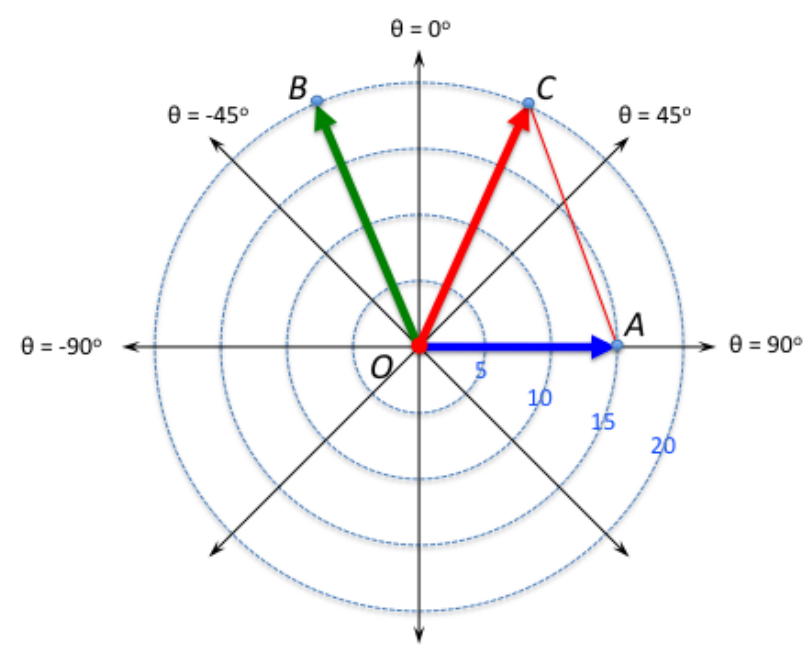

Figure 7. Polar representation of Equation 3 plotted for a range trigger. Standard deviation of wind speed $(\mathrm{OA})$ and standard deviation of relative velocity $(\mathrm{OB})$ add vectorially. The result is standard deviation of atmospheric velocity (OC).

sents the locus of all possible hybrid trigger points, $\mathrm{C}$, as the magnitude of vector $\mathrm{OB}$ is reduced from $20 \mathrm{~m} / \mathrm{s}$ (the range trigger limit) to zero (the velocity trigger limit). In this example, for hybrid triggers with $\sigma_{V_{R}}$ less than $11.2 \mathrm{~m} / \mathrm{s}$, the resulting $\sigma_{V_{A}}$ is smaller than the velocity trigger result. Similarly, the minimum $\sigma_{V_{A}}$ is achieved when $\sigma_{V_{R}}$ is approximately $5.6 \mathrm{~m} / \mathrm{s}$.

\section{Risk Assessment}

In the previous section the negative wind correlation was shown to mitigate the Mach number increase for range triggers. This explains and validates the numerical Monte Carlo results. However, the presence of this correlation creates additional considerations in determining the risk posture of the EDL system. While both velocity and range triggers are affected by dispersions in the wind model, the manner in which they are affected is different. The velocity trigger, by means of a near-zero $\sigma_{V_{R}}$, is affected only by the instantaneous wind at the moment of parachute deploy. Conversely, the range trigger, by means of the wind correlation term, is affected by the integrated history of the winds throughout the entire entry.

This levies stricter requirements on the wind modeling and places an additional lien on simulation results. What if the correlation angle is not realistic or not even present at all? In a worst case, an uncorrelated wind $(\theta=0 \mathrm{deg})$ would increase the estimate of $\sigma_{M}$ in Table 4 from 0.1019 to 0.1178 , raising the estimated $99 \%$-tile Mach number by approximately 0.05 . Using the previous thumb-rule for altitude, this could be countered by an approximate $435 \mathrm{~m}$ decrease in nominal altitude. However, this retuning of the nominal must be weighed against the risk of parachute failure if the Mach increase in simply accepted. Returning to Figure 3, there is little evidence that a 0.05 increase in Mach number would increase 
risk appreciably.

\section{SUMMARY}

A side-by-side comparison of parachute deployment triggers was conducted for MSL. Two triggers, the baseline velocity trigger and an alternate range trigger, were tuned to produce the same nominal parachute deploy Mach number. For each trigger a 6-DoF Monte Carlo analysis was performed using the MSL POST2 end-to-end EDL performance simulation. The results of this study showed that a range trigger has the potential to significantly reduce footprint size with negligible Mach increase and no altitude loss.

An expression was developed, Equation 7, which predicts the standard deviation of Mach number, $\sigma_{M}$ as a function of the three mean values $\left(\mu_{V_{R}}, \mu_{V_{W}}\right.$, and $\left.\mu_{c_{s}}\right)$, three standard deviations $\left(\sigma_{V_{R}}, \sigma_{V_{W}}\right.$, and $\left.\sigma_{c_{s}}\right)$, and two correlation angles $(\theta$ and $\alpha$ ). This expression was shown to have very good agreement with the numerical Monte Carlo results and was used to explain why Mach number failed to increase for the range trigger.

It was shown that a negative correlation coefficient exists between the planet-relative velocity and the wind velocity at parachute deploy. Physically, this correlation exists because the drag, experienced during entry, is a function of the atmospheric-relative velocity, which includes the wind. In the case of a range trigger, this wind correlation mitigates the rise in Mach number uncertainty due to the variance of planet-relative velocity inherent to the trigger.

Finally, Equation 7 was again used to estimate the additional increase in Mach number that could have been observed in the absence of any correlations.

\section{ACKNOWLEDGMENTS}

The author would like to recognize Dick Powell, Jody Davis, Jeremy Shidner, and Alicia Cianciolo of the MSL POST2 Simulation and Flight Dynamics Team for their combined contributions to the development of the POST2 simulation. The author is additionally indebted to Adam Steltzner, Allen Chen, Miguel San Martin, Gavin Mendeck and the rest of the $M S L$ EDL Design Team for their insight, expertise, and sometimes spirited debate on this topic.

\section{REFERENCES}

[1] R. D. Braun, "Mars Exploration Entry, Descent, and Landing Challenges," Journal of Spacecraft and Rockets, Vol. 44, No. 2, pp. 310-323, Mar.-Apr. 2007.

[2] G. L. Carman, D. G. Ives, and D. K. Geller, "ApolloDerived Mars Precision Lander Guidance," AIAA-19984570, AIAA Atmospheric Flight Mechanics Conference and Exhibit, Boston, MA, Aug. 1998.

[3] J. R. Cruz and J. S. Lingard, “Aerodynamic Decelera- tors for Planetary Exploration: Past, Present, and Future" AIAA-2006-6792, AIAA Guidance, Navigation, and Control Conference and Exhibit, Keystone, $\mathrm{CO}$, Aug. 2006.

[4] P. N. Desai, M. Schoenenberger, and F. M. Cheatwood "Mars Exploration Rover Six-Degree-of-Freedom Entry Trajectory Analysis," Journal of Spacecraft and Rockets, Vol. 43, No. 5, pp. 1019-1025, Sep.-Oct. 2006.

[5] C. L. Gillis, "Deployable Aerodynamic Decelerators for Space Missions" Journal of Spacecraft, Vol. 6, No. 8, pp. 885-890, Mar.-Aug. 1969.

[6] R. N. Ingoldby, "Guidance and Control System Design of the Viking Planetary Lander," Journal of Guidance and Control, Vol. 1, No. 3, pp. 189-196, May-Jun. 1978.

[7] G. F. Mendeck and G. L. Carman, "Guidance Design for Mars Smart Landers Using the Entry Terminal Point Controller," AIAA 2002-4502, AIAA Atmospheric Flight Mechanics Conference and Exhibit, Monterey, CA, Aug. 2002.

[8] S. W. Thurman, "Return to the Red Planet: An Overview of the Mars Pathfinder Mission," AIAA-19951534, AIAA Aerodynamic Decelerator Systems Technology Conference, Clearwater Beach, FL, May 1995.

\section{BIOGRAPHY}

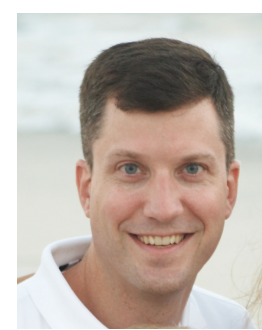

David Way received his Ph.D. in Aerospace Engineering from the Georgia Institute of Technology in 2001. Prior to that, he received a M.S. degree from Georgia Tech in 1997 and a B.S. degree from the United States Naval Academy in 1991, both also in Aerospace Engineering. Following his commission, he served as an officer in the U.S. Navy's nuclear submarine force from 1991 to 1996 . A civil servant assigned to the Atmospheric Flight and Entry Systems Branch of NASA's Langley Research Center, where he has worked since 2001, he is currently serving as LaRC's programmatic POC as well as Simulation and Flight Dynamics Lead for the Mars Science Laboratory mission. In this role, he is responsible for MSL's primary end-to-end EDL performance simulation. 